\title{
Fatigue Crack Growth Under Random Overloads with Retardation
}

\author{
XiaoLi Zou \\ School of Civil Engineering and Architecture, Wuhan Polytechnic University, Wuhan 430023, P. R. China
}

\begin{abstract}
A Monte Carlo simulation scheme is presented to study the behavior of fatigue crack propagation under random overloads. Overloads of Poisson flow with uniform distribution on base-line constant-amplitude cyclic loads are considered. The retardation effect of overload on fatigue crack growth is taken into account by using Wheeler retardation model. The fatigue crack growth curve from initial crack size till fatigue fracture is simulated step by step. With the large number of the simulated samples, the average fatigue crack propagation life is obtained and the influences of overload intensity and magnitude on fatigue lifetime are studied.
\end{abstract}

\section{Introduction}

For reliability design and maintenance plan of structures subjected in service to spectrum loading, statistical prediction of fatigue crack lifetime under random loading is one of the most important problems. However, the main difficulty in evaluating fatigue crack growth in metallic structural components under spectrum loading is associated with the loading interaction effects induced by extreme overload peaks. For engineering structural components, many loading histories can be represented, at first approximation, as a superposition of a constantamplitude cyclic loading and random sequences of overload peaks. As is known, sharp overloads would delay succedent crack growth significantly. To take into account the retardation effect of overload, some stochastic models of fatigue crack growth under random loading have been proposed [1-4]. However, these theoretical models are based on lots of approximate assumptions. On the other hand, it is obviously unrealistic to do a great deal of experiments on fatigue crack growth under random loading. In this paper, the residual stress model proposed by Wheeler [5] is applied to account for retardation effect of overload and random process of crack growth is simulated by Monte Carlo method. Consequently, the statistical characteristics of fatigue crack lifetime under random loading are determined.

\section{Computational model}

Consider a body with a growing fatigue crack, subjected to constant-amplitude cyclic loading with superimposed rare random overloads. It is assumed that the probability of occurrence of overloads is history-independent and that the flow of overloads is stationary. Under these assumptions the number of overloads can be described by a Poisson process characterized by an exponential distribution of inter-overload times with parameter $\lambda$.
The parameter $\lambda$ represents the average number of overloads occurring within one unit time. In addition, the magnitude of the overload stress is also assumed as a random value. Therefore the sequence of overloads is a set of mutually independent random variables with identical probability distribution function.

The residual stress model assumes that due to a high stress concentration at the crack tip a plastic zone is created. The greater the overload peak is, the larger the plastic zone size is. Residual compressive stresses formed in this zone will reduce the magnitude of the tensile stresses during the next load cycle and tend to delay the crack growth.

On the basis of the residual stress concept, Wheeler proposed that the crack propagation rate after the overload is given by

$$
\left(\frac{d a}{d n}\right)_{R}=C_{R} \frac{d a}{d n}
$$

where $C_{R}$ is the retardation coefficient and $d a / d n$ the crack propagation rate under the baseline load. According to Paris-Erdogan equation

$$
\frac{d a}{d n}=c(\Delta K)^{m}
$$

where $c$ and $m$ are material constants, $\Delta K$ the stress intensity factor range.

$$
\Delta K=B \sqrt{\pi a}\left(\sigma_{\max }-\sigma_{\min }\right)
$$

where $B$ is geometric shape parameter, $\sigma_{\max }$ and $\sigma_{\min }$ the maximum stress and minimum stress of the base-line constant-amplitude cyclic loads.

In Eq. 1, the retardation coefficient $C_{R}$ is described by Wheeler as 


$$
C_{R}= \begin{cases}\left(\frac{r_{n}}{a_{p}-a_{n}}\right)^{k} & a_{n}+r_{n} \leq a_{p} \\ 1 & a_{n}+r_{n}>a_{p}\end{cases}
$$

where $k$ is an empirical constant (retardation exponent). As shown in Fig. 1, $a_{0}$ is the crack length at the onset of the overload, $a_{n}$ is the current crack length after the overload ( $a_{n} \geq a_{0}$ ) corresponding to the nth cycle of the baseline load, $\left(a_{p}-a_{0}\right)$ is the radius of the plastic zone produced by the overload, $r_{n}$ is the radius of the current plastic zone corresponding to the nth cycle. The dependence of the size of the plastic zone on the stress intensity factor leads to the following expression

$$
r_{n}=\frac{a_{n}}{A}\left(\frac{A \sigma_{\max }}{\sigma_{y}}\right)^{2}
$$

where $\sigma_{y}$ is the yield strength of the material, parameter $A$ is equal to 1 for plane stress state and 3 for plane strain state.

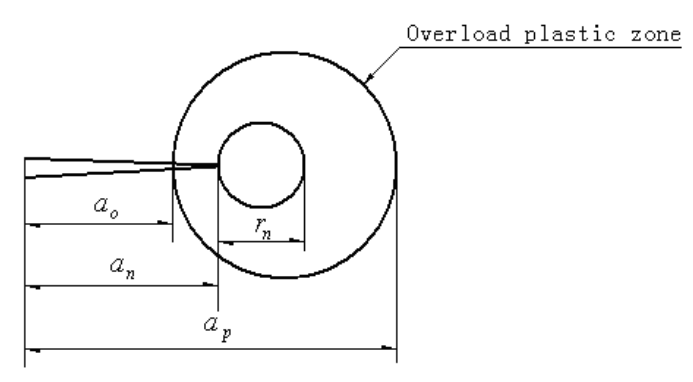

Figure 1. Definition sketch concerning Wheeler equation.

To begin the simulation of crack growth, a series of occurring moment and magnitude of overloads are generated by Monte Carlo method. Then according to above equations, the fatigue crack propagations from initial crack size till final fracture are computed cycle by cycle. In each step of computation, the radius of plastic zone corresponding to the current maximum stress of the base-line cyclic loads or overload stress is calculated from Eq. 5. If the size of the new plastic zone exceeds the old plastic zone, the new plastic zone will replace the old in the next step of computation. As the fatigue crack grows, final fracture will take place once the stress intensity factor $K$ is greater than the fracture toughness of the material $K_{\mathrm{IC}}$. There are two possibilities of final fracture: one is induced by overload, another by stress peak of base-line cyclic loads. Consequently, the fatigue crack lifetime is obtained.

\section{Numerical example and discussion}

Consider propagation of an edge in a semi-infinite body under a random sequence of overloads superimposed on base-line cyclic loading characteristic of minimum stress $\sigma_{\text {min }}=0$ and maximum stress $\sigma_{\max }=300 \mathrm{MPa}$. The random sequence of overloads are characterized by uniform distribution of stress peaks in the range from $\sigma_{o}^{L}$ to $\sigma_{o}^{U}$. In this case the stress intensity factor is $K=1.12 \sigma \sqrt{\pi a}$ and parameter $A=1$ in Eq. 5. The material constants included in calculation are as follow: $\sigma_{y}=1400 \mathrm{MPa} \quad, \quad K_{\mathrm{IC}}=95 \mathrm{MPam}^{1 / 2}, \quad m=3$, $c=9.4 \times 10^{-7}, k=2$.

Assuming initial crack size $a_{i}=2 \mathrm{~mm}$, the fatigue crack propagations from initial crack size till final fracture are computed cycle by cycle for parameters of overload as $\lambda=2 \times 10^{-3}, \sigma_{o}^{L}=450 \mathrm{MPa} \quad$ and $\sigma_{o}^{U}=470 \mathrm{MPa}$. The simulated samples of fatigue crack growth curve are shown in Fig. 2. The dot line in Fig. 2 is crack growth curve without overload and the other three curves with overloads. In the three samples, two final fractures are resulted from peak stress of base-line cyclic loads and the other one from overload. It can be seen from Fig. 2 that the retardation effect of overload is significant.

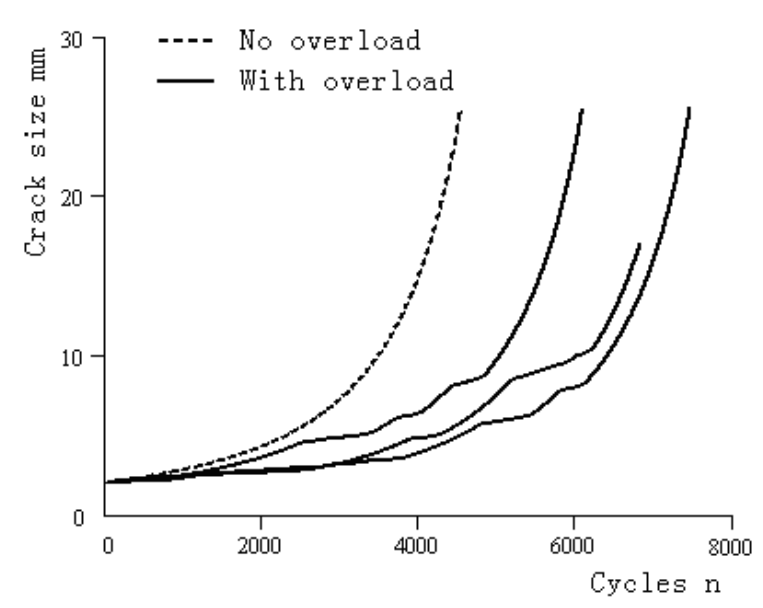

Figure 2. Simulated fatigue crack growth curves.

For studying the influence of overload intensity and magnitude on the fatigue lifetime, 200 samples of fatigue crack growth are computed for every different overload intensity and magnitude. Subsequently, the mean and standard deviation of fatigue lifetimes for various overload intensity and magnitude are obtained, as shown in Table 1. It can be seen from Table 1 that fatigue lifetime increases with overload magnitude. This is because the greater overload is, the more it delays crack growth. Besides, with increase of overload intensity, fatigue lifetime increases at the beginning but decreases afterward. The reason is that the contribution of overload retardation effect to fatigue lifetime is dominant for very small overload intensity but with the increase of overload intensity the possibility of early fracture due to overload increases, which results in decrease of average fatigue lifetime. 
Table 1. The mean and standard deviation of fatigue lifetime for various overload intensity and magnitude.

\begin{tabular}{ccccccccc}
\hline$\left(\sigma_{o}^{L}, \sigma_{o}^{U}\right)$ & \multicolumn{2}{c}{$(400,420)$} & \multicolumn{2}{c}{$(400,450)$} & \multicolumn{2}{c}{$(450,470)$} & \multicolumn{2}{c}{$(450,500)$} \\
\hline$\lambda$ & Mean & Stad Dev. & Mean & Stad Dev. & Mean & Stad Dev. & Mean & Stad Dev. \\
\hline $9 \times 10^{-4}$ & 4757 & 298 & 4860 & 383 & 5275 & 645 & 5590 & 862 \\
$10^{-3}$ & 4800 & 315 & 4889 & 391 & 5406 & 729 & 5576 & 897 \\
$2 \times 10^{-3}$ & 5086 & 418 & 5305 & 563 & 6439 & 911 & 7181 & 1372 \\
$3 \times 10^{-3}$ & 5458 & 437 & 6001 & 584 & 7594 & 1102 & 8555 & 1562 \\
$4 \times 10^{-3}$ & 5755 & 474 & 6429 & 621 & 8762 & 1171 & 10226 & 1683 \\
$5 \times 10^{-3}$ & 6140 & 511 & 6896 & 738 & 9618 & 1407 & 11498 & 1865 \\
$6 \times 10^{-3}$ & 6385 & 576 & 7366 & 812 & 10466 & 1481 & 12132 & 2043 \\
$7 \times 10^{-3}$ & 6698 & 656 & 7790 & 978 & 10738 & 1778 & 12456 & 2671 \\
$8 \times 10^{-3}$ & 6977 & 694 & 8139 & 1117 & 11300 & 2021 & 12706 & 3010 \\
$9 \times 10^{-3}$ & 7200 & 798 & 8461 & 1285 & 11186 & 2425 & 12673 & 3064 \\
$10^{-2}$ & 7289 & 938 & 8562 & 1418 & 11041 & 2773 & 12142 & 3476 \\
$2 \times 10^{-2}$ & 7242 & 1686 & 7881 & 2245 & 8128 & 2556 & 8697 & 2894 \\
\hline
\end{tabular}

\section{References}

1. J. Schijve, ASTM STP. 700, 3 (1980)

2. R. Arone, Engng. Fract. Mech. 39, 895 (1991)

3. R. Arone, Int. J. Fatigue 12, 275 (1990)

4. J. Dominguez, J. Zapatero, B. Moreno, Engng. Fract. Mech. 62, 351 (1999)

5. O. E. Wheeler, Basic Engng. ASME 94, 181 (1972) 